\title{
Tunable Topological Phononic Crystals
}

\begin{abstract}
Ze-Guo Chen and Ying $\mathrm{Wu}^{*}$
Division of Computer, Electrical and Mathematical Sciences and Engineering (CEMSE), King Abdullah University of Science and Technology (KAUST), Thuwal 23955-6900, Saudi Arabia (Received 27 November 2015; revised manuscript received 8 April 2016; published 27 May 2016)

Topological insulators first observed in electronic systems have inspired many analogues in photonic and phononic crystals in which remarkable one-way propagation edge states are supported by topologically nontrivial band gaps. Such band gaps can be achieved by breaking the time-reversal symmetry to lift the degeneracy associated with Dirac cones at the corners of the Brillouin zone. Here, we report on our construction of a phononic crystal exhibiting a Dirac-like cone in the Brillouin zone center. We demonstrate that simultaneously breaking the time-reversal symmetry and altering the geometric size of the unit cell result in a topological transition that we verify by the Chern number calculation and edge-mode analysis. We develop a complete model based on the tight binding to uncover the physical mechanisms of the topological transition. Both the model and numerical simulations show that the topology of the band gap is tunable by varying both the velocity field and the geometric size; such tunability may dramatically enrich the design and use of acoustic topological insulators.
\end{abstract}

DOI: 10.1103/PhysRevApplied.5.054021

\section{INTRODUCTION}

Topological order, a mathematical concept of conserved properties under continuous deformations, was first introduced as a new property of the full energy band along with the discovery of the integer quantum Hall effect (QHE) $[1,2]$. The topological invariant of a two-dimensional (2D) energy band is called the Chern number, a quantity that characterizes the quantized collective behavior of the wave functions of the band. When the sum of the Chern numbers of all the bands below a band gap is not zero, a so-called "topological insulator" emerges. A fascinating property of these topological insulators is that they support a topologically protected one-way propagation edge state without backscattering on their surfaces. Though topological insulators were first observed in electronic systems [3], the concept has been successfully extended to other wave systems because of similarities in the band structures of those systems. The photonic [4-8], elastic [9-19], and mechanical [20-22] analogues of electronic topological insulators have been proposed.

One possible way to achieve a nonzero Chern number is to capitalize on the physics of the QHE and break the timereversal $(\mathcal{T})$ symmetry, which is feasible in photonic crystals by introducing magnetic fields. However, breaking the $\mathcal{T}$ symmetry is fundamentally difficult in acoustic systems because ordinary acoustic materials generally conserve their $\mathcal{T}$ symmetry. Very recently, Alù and co-workers [23] tackled this problem by introducing a rotation flow in the background field to break the $\mathcal{T}$ symmetry in an acoustic system. Later,

\footnotetext{
*Corresponding author. ying.wu@kaust.edu.sa
}

their solution was generalized to phononic crystals to achieve acoustic topological insulators $[9,10,14,23]$.

Most of the progress in this area $[9,10,14]$ was made with lattices with $C_{6 v}$ symmetry in which there exists a Dirac cone protected by the $\mathcal{T}$ symmetry at the $K$ point of the Brillouin zone. The existence of the Dirac cone is attributed to the symmetry of the lattice; it is not affected by the geometric size of the inclusions in the crystal [24]. Because of the linear dispersion of the Dirac cone, breaking the $\mathcal{T}$ symmetry may open a nontrivial band gap and introduce edge states [25]. This process can be described by a two-band model [2]. Recently, another kind of linear dispersion, namely, a Diraclike cone, was found at the center of the Brillouin zone of lattices with both $C_{6 v}$ and $C_{4 v}$ symmetries [26,27]. Different from Dirac cones, Dirac-like cones arise from accidental degeneracy of a single mode and a doubly degenerate mode and are sensitive to the geometric size of the inclusion. They are, therefore, protected by both the $\mathcal{T}$ symmetry and the particular geometric parameters of the crystal. We wonder what the consequences will be, in particular, how the three bands associated with the Dirac-like cone evolve and what the topological invariant is, if breaking the symmetry and altering the geometric size of the crystal occur simultaneously. A comprehensive understanding of the degrees of freedom needed to modulate the topological invariant in such systems will provide a physical picture of the mechanisms of the band-gap opening at the Brillouin zone center, further expanding our ability to tailor acoustic waves.

To thoroughly investigate this problem, we construct a 2D phononic crystal (PC) with $C_{4 v}$ symmetry. By carefully adjusting the size of the crystal's building block, we find a Dirac-like cone in its band structure associated with a triply degenerate state at the Brillouin zone center. We observe that 
the degeneracy can be lifted by altering the geometrical parameters of the inclusion or by introducing airflow, and both ways can introduce a band gap at the frequency of the degeneracy. We develop a tight-binding model to systematically study the underlying mechanisms of the bandgap opening, which reveals that altering the geometry of the inclusion is equivalent to changing the coupling coefficients between the neighboring lattices and that introducing airflow indicates a Zeeman-type perturbation of the on-site energy. By calculating the Chern numbers of the bands, we find that the induced band gaps exhibit different topologies. Purely changing the geometric size of the inclusion does not give rise to nontrivial band gaps, whereas introducing airflow does. We further explore the system by combining the two ways of breaking the degeneracy. A somewhat unexpected result is that breaking the $\mathcal{T}$ symmetry is not sufficient to yield a nontrivial band gap if the geometric size of the inclusion is below a certain critical point. This critical point is called the topological transition point, and its value depends on the intensity of the introduced airflow. Such a topological transition behavior can also be captured by the tight-binding model in conjunction with a perturbation theory, which gives a Hamiltonian similar to the quantum anomalous Hall model [28].

Based on this interesting finding, we demonstrate, with numerical experiments, the existence of a tunable state in phononic crystals. When the geometric size of the inclusion or the intensity of the airflow exceed the topological transition point, the state changes from a localized state (attributed to the trivial band gap) into a topology-protected one-way edge state (a result of the nontrivial band gap) that is immune to backscattering.

\section{MODEL AND RESULTS}

\section{A. Sample}

The 2D PC considered here is composed of a square array of acoustic waveguides. The unit cell is a hollow ring attached by four subwavelength rectangular waveguides as illustrated in Fig. 1(a). The lattice constant is $a=2 \mathrm{~m}$, and the inner and outer radii of the ring are $r_{0}=0.35 \mathrm{~m}$ and $r_{1}=0.5 \mathrm{~m}$, respectively. The width of the rectangular waveguide is $d$, which can be adjusted. Inside the ring, the air flows counterclockwise (we assume that the flow is confined within the ring). The acoustic wave propagation in such a structure is protected by the $\mathcal{T}$ symmetry if there is no airflow, but it behaves according to the following equation if an irrotational airflow is introduced [29]:

$$
\begin{aligned}
& -\frac{\rho}{c^{2}} i \omega(i \omega \phi+\vec{v} \cdot \nabla \phi) \\
& \quad+\nabla \cdot\left(\rho \nabla \phi-\frac{\rho}{c^{2}}(i \omega \phi+\vec{v} \cdot \nabla \phi) \vec{v}\right)=0,
\end{aligned}
$$

where $\phi$ represents the velocity potential, $\rho$ and $c$ denote the mass density and the velocity of sound in air, respectively, $\omega$ is the angular frequency of the acoustic wave, and $\vec{v}$ is the velocity field of the airflow.

We start with a PC without the introduction of airflow. The band-structure calculation performed by COMSOL Multiphysics (a finite-element software) shows that there are three eigenmodes that degenerate at the $\Gamma$ point when the width of the waveguide $(d)$ equals $0.07336 \mathrm{~m}$ and a Dirac-like cone appears. [See the blue curve in Fig. 1(b)]. The corresponding pressure-field distributions are shown in
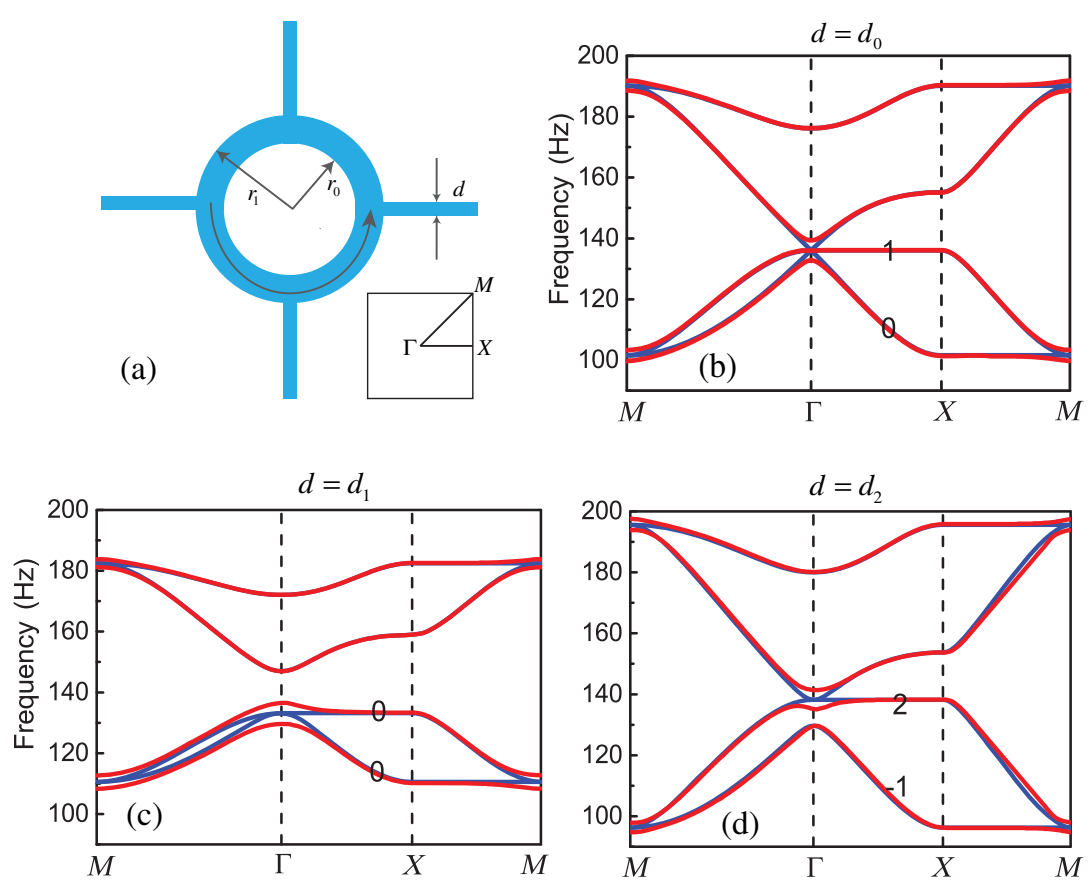

FIG. 1. (a) Schematics of a unit cell of our phononic crystal. The ring is connected by rectangular waveguides. The lower inset illustrates the reciprocal lattice. (b)-(d) Band structure of the phononic crystal whose rectangular waveguide has a width of $d_{0}=0.07336 \mathrm{~m}$, $d_{1}=0.04 \mathrm{~m}$, and $d_{2}=0.1 \mathrm{~m}$ without an airflow (shown in blue curves) and with a circulating airflow at $v=10 \mathrm{~m} / \mathrm{s}$ (shown in red curves). When airflow is introduced, the Chern numbers are marked on their corresponding bands under the band gap. 

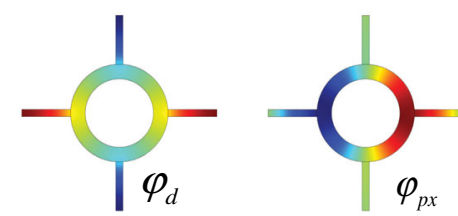

(a)
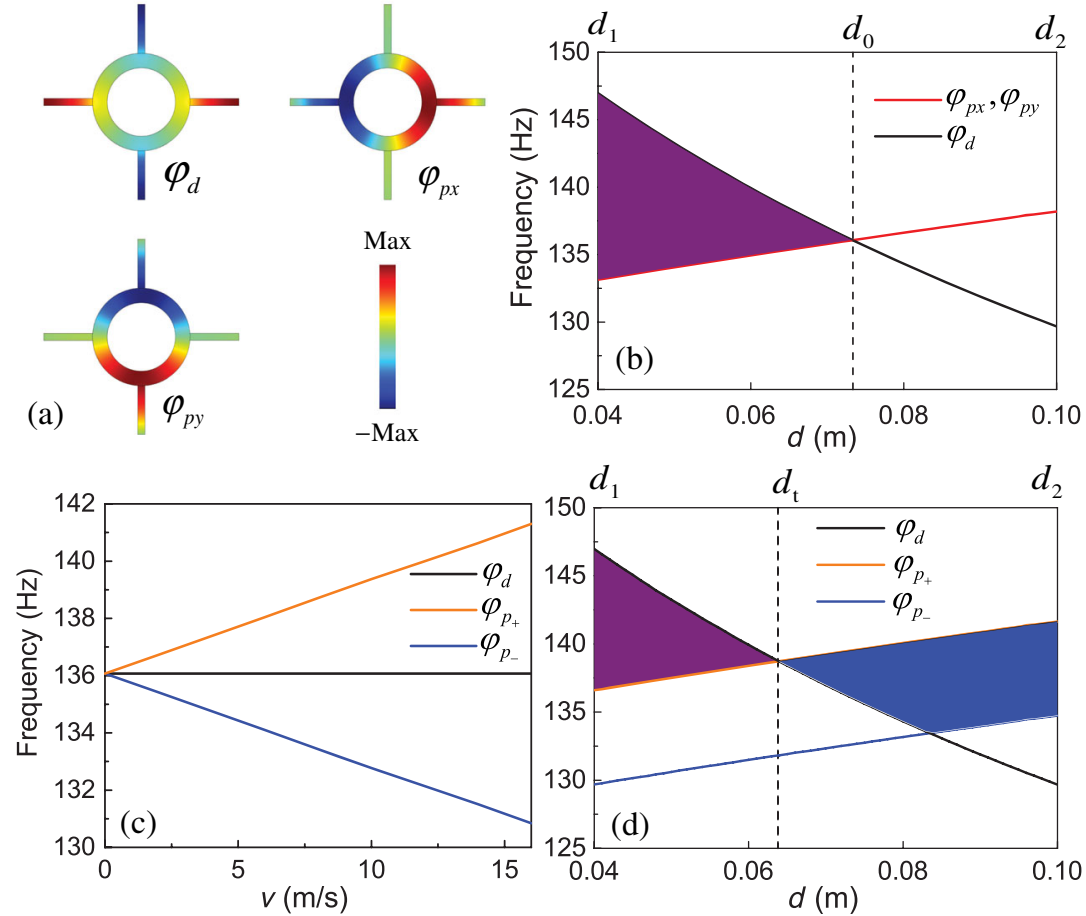

FIG. 2. (a) Pressure-field distributions of three Bloch eigenstates at $\Gamma$ point marked as $\varphi_{d}, \varphi_{p x}$, and $\varphi_{p y}$. Dark red and dark blue indicate the positive and negative maxima. (b) The eigenfrequency of the eigenstates varies as functions of $d$ when no airflow is introduced. The black and red curves correspond to the eigenfrequencies of $\varphi_{d}$ and $\varphi_{p x}\left(\varphi_{p y}\right)$ eigenstates, respectively. Purple area indicates the system has a complete band gap. (c) The eigenfrequency of $\varphi_{d}, \varphi_{p_{+}}$, and $\varphi_{p_{-}}$versus the velocity field of the induced airflow. (d) The same as (b) but an airflow with $v=10 \mathrm{~m} / \mathrm{s}$ is introduced. The purple and blue areas indicate the regions with a trivial and nontrivial band gap, respectively.

Fig. 2(a); two of these distributions share a similar pattern and are marked in the figure as $\varphi_{p x}$ and $\varphi_{p y}$, and the third one is marked as $\varphi_{d}$. It is known that the degeneracy of the three states is accidental [26] because $\varphi_{d}$ deviates from $\varphi_{p x}$ and $\varphi_{p y}$ if the width of the waveguide changes. Because of the $C_{4 v}$ symmetry at the $\Gamma$ point, $\varphi_{p x}$ and $\varphi_{p y}$ always degenerate, regardless of the size of the waveguide. Shown in the blue curves in Figs. 1(c) and 1(d) are the band structures for PCs with $d_{1}=0.04 \mathrm{~m}<d_{0}$ and $d_{2}=0.1 \mathrm{~m}>d_{0}$, respectively. The eigenfrequency of the doubly degenerate state is lower (higher) than that of the single state if the width is smaller (larger) than $d_{0}$. We plot in Fig. 2(b) the eigenfrequencies of the single state $\varphi_{d}$ and the doubly degenerate state $\varphi_{p x}$ and $\varphi_{p y}$ as functions of the width of the waveguide and find an intersection point at $d_{0}$ indicating the occurrence of the accidental degeneracy of the three states. The band structures shown in Figs. 1(b)-1(d) exhibit that for a PC with narrow waveguides, there exists a complete band gap. For example, a band gap exists in the 133.1- to $147.0-\mathrm{Hz}$ frequency range when $d=d_{1}$. It closes at $d=d_{0}$, but no complete band gap appears when the waveguide becomes wider, even though the accidental degeneracy is lifted and there is a directional band gap as shown in Fig. 1(d). We also notice two doubly degenerate points in the corner of the Brillouin zone, i.e., the $M$ point. They always degenerate even as $d$ changes and, therefore, are deterministic.

\section{B. Theoretical model}

To understand better the behavior of the abovementioned band structures, we develop a tight-binding model to describe the associated dispersion relation $[30,31]$. We note that the eigenmodes at the $\Gamma$ point are calculated with the periodic boundary condition imposed. If a unit cell is placed in the free space, modes with similar symmetries denoted as $\Phi_{p x}, \Phi_{p y}$, and $\Phi_{d}$ also exist. By taking the symmetry of these free-space modes into account, we write the kernel of the Hamiltonian in the basis of state $\Phi_{d}, \Phi_{p y}$, and $\Phi_{p x}$ (denoted as $|d\rangle,|p y\rangle$, and $|p x\rangle$ ) as follows:

$$
H_{0}=\left[\begin{array}{ccc}
E_{d}+2 t_{x}^{11}\left(\cos k_{x} a+\cos k_{y} a\right), & -2 i t_{x}^{12} \sin k_{y} a, & 2 i t_{x}^{12} \sin k_{x} a, \\
2 i t_{x}^{12} \sin k_{y} a, & E_{p y}+2 t_{x}^{22} \cos k_{y} a+2 t_{y}^{22} \cos k_{x} a, & 0, \\
-2 i t_{x}^{12} \sin k_{x} a, & 0, & E_{p x}+2 t_{y}^{22} \cos k_{y} a+2 t_{x}^{22} \cos k_{x} a
\end{array}\right],
$$

where $E_{d}, E_{p x}$, and $E_{p y}$ are the on-site energy of the rings, and $t_{m}^{i j}=\left\langle\Phi_{i}(\vec{r})|H| \Phi_{j}\left(\vec{r}+\vec{r}_{m}\right)\right\rangle$ represents different types of first-neighbor coupling coefficients between the above-mentioned free-space modes. Here, $\Phi_{i}(i=1,2,3)$ correspond to $\Phi_{d}, \Phi_{p y}$, and $\Phi_{p x}$, respectively. $\vec{r}_{m}(m=x, y)$ are lattice vectors. Equation (2) is simplified by considering the symmetry, for example, $t_{x}^{22}=t_{y}^{33}$. Both the on-site energy and the coupling coefficients are functions of the 
sizes of the rings and the rectangular waveguides. The wave functions inside the waveguide contribute to the coupling coefficient, which is, therefore, a function of the width of the waveguide. At $\Gamma$ point $\left(k_{x}=k_{y}=0\right)$, Eq. (2) becomes a diagonal matrix, and its diagonal terms are nothing but the eigenvalues of the Hamiltonian, which are $E_{s}+4 t_{x}^{11}$, $E_{p y}+2 t_{x}^{22}+2 t_{y}^{22}$, and $E_{p x}+2 t_{x}^{22}+2 t_{y}^{22}$. These eigenvalues are proportional to the eigenfrequencies of the modes $\varphi_{d}, \varphi_{p y}$, and $\varphi_{p x}$, respectively. The on-site energy does not change due to the fixed size of the ring. The coupling coefficients are proportional to the width of the rectangular waveguide because the waveguide is narrow enough, and it supports only the fundamental waveguide mode. We can, therefore, expect that the eigenvalues of the Hamiltonian (or the eigenfrequencies of the three modes) depend linearly on the width of the waveguide, which qualitatively agrees with the numerical simulations presented in Fig. 2(b). At $d=d_{0}$, all the eigenvalues of the Hamiltonian become identical. Both the tight-binding model and the simulation of the real systems suggest that $d_{0}$ is a transition point, as there exists only a band gap when $d<d_{0}$. This band gap is topologically trivial as the $\mathcal{T}$ symmetry is preserved.

When airflow is introduced, the $\mathcal{T}$ symmetry breaks. Here, for simplicity but without loss of generality, we choose the following velocity field distribution $[10,14]$ :

$$
\vec{v}(x, y)=\left(\frac{-v y}{\sqrt{x^{2}+y^{2}}}, \frac{v x}{\sqrt{x^{2}+y^{2}}}\right)=\vec{e}_{\theta},
$$

where $\vec{e}_{\theta}$ is the azimuthal unit vector along the counterclockwise direction, and $v$ is the amplitude of the velocity field. The advantage of choosing such a velocity field distribution lies in the constant radial component of $\vec{v}$. The velocity field is invariant under time reversal, and it is not difficult to see that the system, as well as its describing equation [Eq. (1)], is no longer symmetric to the time reversal. As a result, the doubly degenerate state of $\varphi_{p x}$ and $\varphi_{p y}$ split and so do their corresponding on-site energy. From the field patterns shown in Fig. 2(a), where there is no airflow, we find that the $\varphi_{p x}$ and $\varphi_{p y}$ modes are mainly from the resonance inside the ring when the circumference of the ring approximately equals the wavelength, whereas the $\varphi_{d}$ mode is mainly from the resonance inside the rectangular waveguide. Intuitively, circulating airflow will not affect the eigenfrequency of $\varphi_{d}$ by much, but it will change the eigenfrequencies of $\varphi_{p x}$ and $\varphi_{p y}$. In fact, it changes the eigenfrequencies of linear combinations of $\varphi_{p x}$ and $\varphi_{p y}$, i.e., $\varphi_{p \pm}=\left(\varphi_{p y} \pm i \varphi_{p x}\right) / \sqrt{2}$. The choice of $\varphi_{p \pm}$ is consistent with the change of the symmetry from a $C_{4 v}$ point group into a $C_{4}$ point group when the $\mathcal{T}$ symmetry is broken. According to the superposition principle, the acoustic wave circulates inside the ring at different velocities, $c+v$ and $c-v$, which leads to the splitting of the resonance frequencies as follows: $\omega_{ \pm}=(c \pm v) / R_{\mathrm{av}}$, where $R_{\mathrm{av}}=\left(r_{0}+r_{1}\right) / 2$ is the average of the inner and outer radii, and $\omega_{0}=c / R_{\mathrm{av}}$ is the eigenfrequency of the degenerated modes $\varphi_{p x}$ and $\varphi_{p y}$ without the airflow [32]. We use COMSOL to calculate the eigenfrequencies of the three modes and plot them in Fig. 2(c) as functions of the velocity field. This plot indeed shows the linear behavior in the splitting of the eigenfrequencies of $\varphi_{p+}$ and $\varphi_{p-}$ and the almost constant eigenfrequency of $\varphi_{d}$ as $v$ increases, supporting our analysis. Therefore, the external velocity field may be viewed as an analogue to a magnetic vector field that gives rise to the Zeeman effect. In the following, we incorporate the external velocity field into the tightbinding model that we derived earlier to explore the characteristics of the system without the $\mathcal{T}$ symmetry.

Considering the external velocity field, we write the new Hamiltonian as

$$
H=H_{0}+H_{z}
$$

where $H_{z}$ represents the Zeeman-type Hamiltonian induced by the external velocity field on the basis of $|d\rangle,\left|p_{+}\right\rangle$, and $\left|p_{-}\right\rangle$. As mentioned before, the external velocity mostly couples to the $\Phi_{p y}$ and $\Phi_{p x}$ modes, while it almost does not couple to the $\Phi_{d}$ mode, and we superpose the $\Phi_{p y}$ and $\Phi_{p x}$ modes linearly and construct a new basis $\left|p_{ \pm}\right\rangle=$ $(|p y\rangle \pm i|p x\rangle) / \sqrt{2}$. Under the basis of $|d\rangle,\left|p_{+}\right\rangle$, and $\left|p_{-}\right\rangle$, the Zeeman-type Hamiltonian is written as

$$
H_{z}=\left[\begin{array}{ccc}
0 & 0 & 0 \\
0 & \Delta z & 0 \\
0 & 0 & -\Delta z
\end{array}\right]
$$

where $\Delta z$ is the coupling constant and depends on the external velocity field. We rewrite the $H_{0}$ by using basis transformation and get the total Hamiltonian

$$
H=\left[\begin{array}{ccc}
E_{d}(\vec{k}), & \sqrt{2} t_{x}^{12}\left(\sin k_{x} a-i \sin k_{y} a\right), & -\sqrt{2} t_{x}^{12}\left(\sin k_{x} a+i \sin k_{y} a\right), \\
\sqrt{2} t_{x}^{12}\left(\sin k_{x} a+i \sin k_{x} a\right), & \frac{1}{2}\left[E_{p y}(\vec{k})+E_{p x}(\vec{k})\right]+\Delta z, & \frac{1}{2}\left[E_{p y}(\vec{k})-E_{p x}(\vec{k})\right], \\
-\sqrt{2} t_{x}^{12}\left(\sin k_{x} a-i \sin k_{y} a\right), & \frac{1}{2}\left[E_{p y}(\vec{k})-E_{p x}(\vec{k})\right], & \frac{1}{2}\left[E_{p y}(\vec{k})+E_{p x}(\vec{k})\right]-\Delta z
\end{array}\right],
$$


where $E_{d}(\vec{k}), E_{p y}(\vec{k})$, and $E_{p x}(\vec{k})$ are diagonal terms of the original $H_{0}$ under the old basis. Near the point, we consider the case when $E_{d}(0)$ is much closer to $\left[E_{p y}(0)+\right.$ $\left.E_{p x}(0)\right] / 2+\Delta z$ than $\left[E_{p y}(0)+E_{p x}(0)\right] / 2-\Delta z$. This condition makes it possible to project out the $\left|p_{-}\right\rangle$state by a perturbation theory and deduce the following reduced Hamiltonian under the basis of $\left(|d\rangle,\left|p_{+}\right\rangle\right)$to the linear term in $\vec{k}$,

$H_{A}=\left[\begin{array}{cc}E_{d}(0), & \sqrt{2} i t_{x}^{12}\left(k_{y} a+i k_{x} a\right), \\ -\sqrt{2} i t_{x}^{12}\left(k_{y} a-i k_{x} a\right), & \frac{1}{2}\left[E_{p y}(0)+E_{p x}(0)\right]+\Delta z\end{array}\right]$.

The details can be found in the Appendix. Equation (6) is a typical description of the quantum anomalous Hall (QAH) model [28] and indicates a topological transition occurring at $E_{d}(0)=\left[E_{p y}(0)+E_{p x}(0)\right] / 2+\Delta z$.

Since $E_{d}(0), E_{p y}(0)$, and $E_{p x}(0)$ depend on the waveguide width $d$, and $\Delta z$ is a function of external velocity field $v$, the occurrence of the topological transition should depend on both quantities. Figure 3 illustrates a phase diagram of the topological property of the band gap as a function of waveguide width and the strength of the external velocity field. The line in the middle represents the topological transition. Figure 3 indicates that the topology of the band gap is tunable by modulating $d$ and $v$. This fact will benefit the design of tunable topological insulators.

To verify the conclusion we draw from the tight-binding model with broken $\mathcal{T}$ symmetry, we introduce an airflow at $v=10 \mathrm{~m} / \mathrm{s}$ into the systems that have been presented in Figs. 1(b)-1(d) and plot the corresponding band structures as red curves in the same figures. As expected, the branch associated with $\varphi_{d}$ is mostly unaffected, and the branches associated with $\varphi_{p x}$ and $\varphi_{p y}$ do not degenerate at the $\Gamma$ point. Consequently, complete band gaps open for the cases

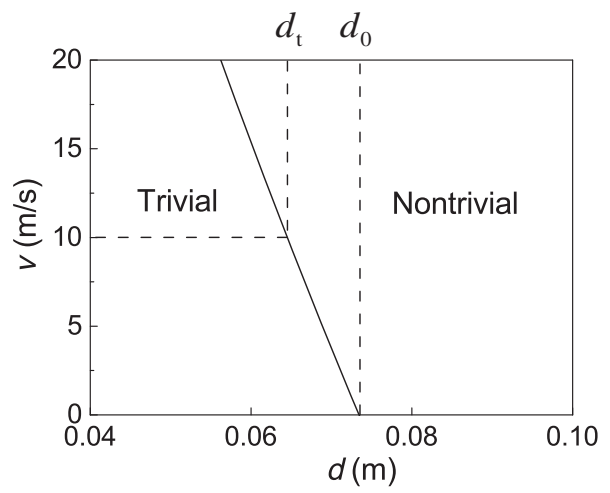

FIG. 3. Phase diagram of the topology property of the band gap with combined modulation of the width of the waveguide and the intensity of the airflow. Topological transition occurs across the solid line. when $d=d_{0}$ [Fig. 1(b)] and $d=d_{2}$ [Fig. 1(d)]. For the case of $d=d_{1}$ [Fig. 1(c)], introducing the airflow reduces only the range of the band gap from 133.1 and $147.0 \mathrm{~Hz}$ to 138.2 and $141.3 \mathrm{~Hz}$ without opening a new band gap. Meanwhile, the degeneracies at the $M$ point are lifted for all three cases because of the broken $\mathcal{T}$ symmetry. The changes in the band structures after introducing the airflow suggest that there will be a topological transition point at $d=d_{t}$ for a particular $v$. The lifted degeneracy by introducing the airflow will (or will not) lead to a new band gap when $d \geq d_{t}$ (or $d<d_{t}$ ). To better demonstrate the transition process, we plot in Fig. 2(d) the eigenfrequencies $\varphi_{d}$ and $\varphi_{p \pm}$ calculated by COMSOL as functions of waveguide width. It is not difficult to see that the eigenfrequency of $\varphi_{d}$ is the same as that of $\varphi_{p+}$ at $d_{t}$, across which band inversion of $\varphi_{p+}$ and $\varphi_{d}$ occurs. This band inversion is a consequence of combined changes of width of the waveguide and the intensity of the airflow. Here, we point out that $d_{t}$ is different from $d_{0}$, as $d_{t}$ corresponds to the point at which the two eigenvalues of $H_{A}$ [Eq. (6)] are identical, and $d_{0}$ is the point at which the three eigenvalues of the original Hamiltonian without the airflow [Eq. (2)] are the same. It can be easily proven that $d_{t}<d_{0}$.

The band-inversion process predicted by Eq. (5) is verified numerically. In the following, we affirm this band inversion is associated with a topological transition. To do this, we need to evaluate the "gap Chern number," a topological invariant that can characterize a band gap. A general way to calculate this invariant is to sum the Chern numbers, which are expressed by the following equation of all bands below the band gap:

$$
C=\frac{i}{2 \pi} \sum_{n} \int_{B Z} \nabla_{\vec{k}} \times\left(\left\langle u_{n}(\vec{k})\left|\nabla_{\vec{k}}\right| u_{n}(\vec{k})\right\rangle\right) d^{2} \vec{k},
$$

where $u_{n}(\vec{k})$ is the Bloch function of the $n$th band at $\vec{k}$. It is possible to substitute the numerically simulated eigenstates on the band into Eq. (7) to compute the Chern number, but this is computationally challenging. It is also possible to use the Hamiltonian including the airflow [Eq. (5)], solve for the eigenvectors, and plug them into Eq. (7). However, the vector potential is dependent on the frequency [9], which makes the calculation of the Chern number over the whole Brillouin zone difficult because the Hamiltonian is valid only near the high symmetry points. Fortunately, only the region near the broken degeneracy points generates a nonvanishing "Berry flux," which contributes to the band's Chern number [33], indicating that we can construct Hamiltonians at the points of degeneration to determine the Chern numbers of the four bands. This method is utilized in determining the Chern number of the bands connected by Dirac cones in phononic crystals with broken $\mathcal{T}$ symmetry and with $C_{3 v}$ or $C_{6 v}$ symmetry [10]. Recently, the Chern number was found to be constrained by the symmetry properties of the eigenstates at high symmetry points [34]. 
TABLE I. Eigenvalues of the symmetry operators of eigenstates at different high symmetry points and resulting contributions to the gap Chern numbers. Subscripts 1 and 2 correspond to the bands with eigenfrequencies from low to high.

\begin{tabular}{lrrr}
\hline \hline & $d_{1}$ & $d_{0}$ & $d_{2}$ \\
\hline$\hat{C}_{4} \Gamma_{1}$ & $-i$ & $-i$ & -1 \\
$\hat{C}_{4} M_{1}$ & $-i$ & $-i$ & $-i$ \\
$\hat{C}_{2} Y_{1}$ & -1 & -1 & -1 \\
$\hat{C}_{4} \Gamma_{2}$ & $i$ & -1 & $-i$ \\
$\hat{C}_{4} M_{2}$ & $i$ & $i$ & $i$ \\
$\hat{C}_{2} Y_{2}$ & -1 & -1 & -1 \\
$C_{\text {gap }}$ & 0 & 1 & 1 \\
\hline \hline
\end{tabular}

The point group of $\vec{k}$ at the $\Gamma$ point becomes $C_{4}$ when the airflow is introduced. We consider all the $C_{m}$-invariant points below the band gap in a $C_{n}$-invariant insulator for each $m$ dividing $n$. The gap Chern number modulo $n$ is related to the eigenvalues of corresponding $C_{m}$ operators of all these points:

$$
i^{C}=\prod_{j} \xi_{j}(\Gamma) \xi_{j}(M) \zeta_{j}(Y),
$$

where $j$ is the label of bands under the band gap, $\xi$ and $\zeta$ are the respective eigenvalues of the $\hat{C}_{4}$ and $\hat{C}_{2}$ operators on the eigenstates at high symmetry points. Table I presents the calculated results from Eq. (8) indicating the various topological properties of the band gaps. The band gaps with different Chern numbers are highlighted in different colors in Fig. 2(d) and marked by numbers in Figs. 1(b)-1(d). From Fig. 2(d), we find that the Chern number remains zero when $d$ is smaller than $d_{t}$ even with broken $\mathcal{T}$ symmetry, and it changes to one when $d$ is larger than $d_{t}$. When $d$ becomes even larger, a band inversion between $\varphi_{d}$ and $\varphi_{-}$states also occurs, but it does not contribute to the gap Chern number. The results on the Chern number calculation support the prediction of the tight-binding model and clearly suggest the existence of a topological transition that occurs by modulating the geometric parameters of the inclusion in a system with broken $\mathcal{T}$ symmetry, which renders additional freedom to manipulate the gap Chern number in the $2 \mathrm{D}$ case.

\section{Examples and discussion}

Insulators with non-zero gap Chern numbers are topologically nontrivial. A property in such insulators is the presence of a gapless edge state between gaps with different topological invariants [1]. The band structure of an $8 \times 1$ supercell with $d=0.1 \mathrm{~m}$ and $v=10 \mathrm{~m} / \mathrm{s}$ is calculated to confirm the existence of such gapless edge states. This supercell is infinite along the $x$ direction and is terminated by rigid boundaries (topologically trivial) in the $y$ direction. Figure 4(a) shows the band structure of such a supercell. It

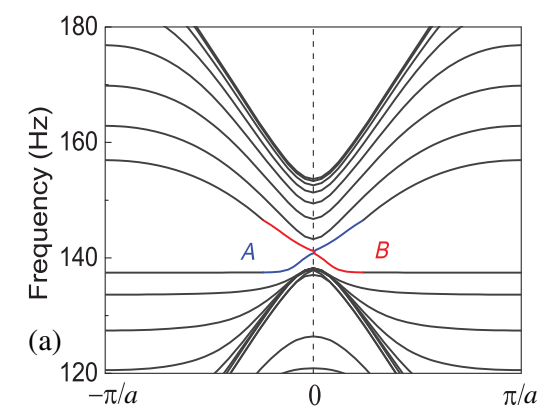

(b)
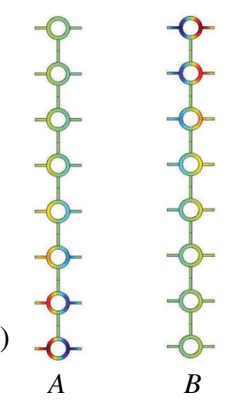

FIG. 4. (a) Band structure of a $1 \times 8$ supercell $\left(d=d_{2}\right.$, $v=10 \mathrm{~m} / \mathrm{s}$ ). Dispersion relations of the edge states are shown in colored curves, and the bulk bands are plotted in gray curves. Red and blue curves represent different edge modes. (b) The pressure-field distribution of edge modes for $A, k=-0.1 \pi / a$ and $B, k=0.1 \pi / a$.

exhibits one one-way edge mode, which agrees with the gap Chern numbers. The dispersion relation highlighted in blue (red) marked as $A(B)$ is associated only with modes having a positive (negative) group velocity. At a given frequency, the modes corresponding to the $A$ and $B$ branches are confined on the opposite edges of the supercell as shown in Fig. 4(b). The $A$ and $B$ branches support modes bounded on the bottom and top edges, respectively. This property leads to one-way propagation at the edge. At some particular frequencies located inside the gap region of the bulk mode, there exists only an edge mode, implying that there is no backward scattering into the bulk. The features exhibited in Figs. 4(b) and 4(b) ensure the existence of topologically protected one-way propagation of the edge modes.

To demonstrate the existence of acoustic one-way edgemode propagation, we perform finite-element simulations of some finite-sized samples. The first sample is composed of $8 \times 24$ unit cells as shown in Fig. 5(a). The upper, bottom, and right boundaries of the sample are hard walls that can be treated as insulators with zero Chern numbers. A plane-wave radiation condition is set on the left boundary. A point source with frequency $139 \mathrm{~Hz}$, a common band-gap frequency for various widths $d$ is located in the bottom boundary. Also, in the bottom boundary there is a defect introduced by removing the airflow inside the ring, which is marked as a green circle in Fig. 5(a). The pressurefield distribution unambiguously shows that the sound wave propagates counterclockwise and circumvents the defect without backscattering.

Changing the topological invariant by varying the geometry also allows us to create the interface states. Another finite-sized sample with $8 \times 25$ lattices is presented in Fig. 5(b). Its upper half contains $4 \times 25$ lattices in which the width of the waveguide is $d_{1}$; its lower half contains the other lattices in which the width of the waveguide is changed into $d_{2}$. A point source is located on the interface between the upper and lower halves. The 
(a)

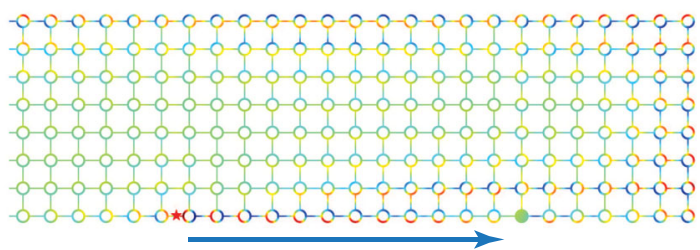

(b)

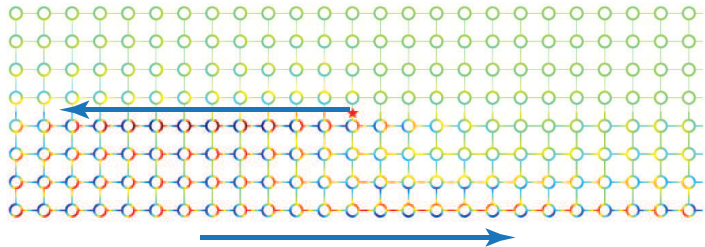

FIG. 5. Demonstration of properties resulting from nontrivial band gaps by tuning the geometric size of the phononic crystal. The source indicated by a star is a point source at $139 \mathrm{~Hz}$. (a) Topologically protected one-way propagation that is immune to defects (marked by a green circle) and without backscattering. Here, the width of the waveguide is $d=d_{2}$ and the velocity of the airflow is $v=10 \mathrm{~m} / \mathrm{s}$. (b) One-way interface state propagation on the interface between two different lattices. The width of the waveguide is $d=d_{1}$ in the upper half and $d=d_{2}$ in the lower half. The arrows in (a) and (b) indicate the directions of propagation of the edge or interface mode.

frequency remains at $139 \mathrm{~Hz}$. According to our previous analysis, this frequency is located inside the band gaps of both the upper and lower lattices. The band gap is trivial for the upper lattices and nontrivial for the lower lattices. This means that no energy will penetrate into the upper lattices and that there should be an interface state. The pressure field shown in Fig. 5(b), indeed, demonstrates the existence of the interface state and the unidirectional behavior of the propagation of the sound wave due to the different topologies of the band gaps of the upper and lower lattices.

As supported by the results shown in Fig. 5, the topology of the band gap can be tuned by changing the geometry of the PC. On the other hand, according to the tight-binding model and symmetry analysis presented earlier, varying the velocity field is another effective way to tune the topology of the band gap. In Fig. 6(a), we plot the eigenfrequencies of $\varphi_{d}$ and $\varphi_{p_{+}}$ as functions of the velocity field for a lattice with a fixed waveguide width, i.e., $d=0.065 \mathrm{~m}$. Similar to Fig. 2(d), we use purple and blue to indicate the regions for trivial band gaps and nontrivial band gaps, respectively. We compare the sound-wave propagation in two finite-sized samples. They share the same geometric configuration and are excited by the same source, but they are exposed to distinct airflows. The one with velocity field $v=5 \mathrm{~m} / \mathrm{s}$ [Fig. 6(b)] exhibits typical trivial band-gap behavior as the acoustic pressure field is almost localized around the source, whereas the other one with velocity field $v=15 \mathrm{~m} / \mathrm{s}$ [Fig. 6(c)] exhibits one-way edge states propagating counterclockwise.
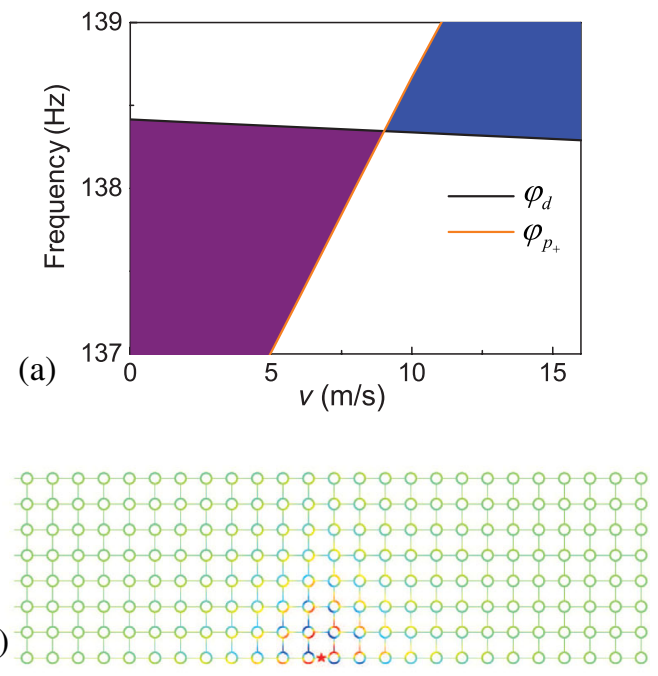

(c)

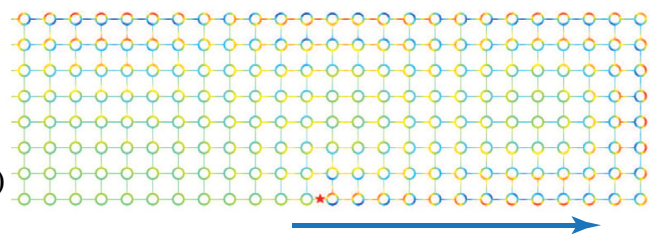

FIG. 6. (a) The eigenfrequencies of the eigenstates vary as functions of $v$. Black and red curves correspond to the $\varphi_{d}$ and $\varphi_{p_{+}}$ modes, respectively. The purple and blue areas indicate the region of the band gap with a different topological invariant, where the intersection is the topological transition point. (b) The simulated pressure-field distribution excited by a point source in a phononic crystal with airflow ( $v=5 \mathrm{~m} / \mathrm{s})$. (c) The same as (b), but the velocity field of the airflow is $v=15 \mathrm{~m} / \mathrm{s}$. In both cases, the rectangular waveguide in the phononic crystal has a width of $d=0.065 \mathrm{~m}$. The source has a frequency of $f=138.4 \mathrm{~Hz}$ and is marked by a star. The arrow indicates the direction of propagation of the edge mode.

\section{CONCLUSION}

In conclusion, we report on our design of a tunable PC that exhibits topologically nontrivial band gaps by breaking the $\mathcal{T}$ symmetry and modulating the geometric parameters of the inclusion. The mechanisms that lead to band-gap opening by breaking the $\mathcal{T}$ symmetry and by varying the geometric parameters of the inclusion as well as their interplay are studied systematically by using a tightbinding model, a rigorous symmetry analysis, and numerical simulations. We find a topological transition point that is related to both $\mathcal{T}$ symmetry and the geometric size of the inclusion, which suggests that the topology can be changed by tuning the strength of the velocity field and/or the size. The transition from a localized state to a robust oneway propagated edge mode is verified by our numerical experiments. Our findings can inspire designs of acoustic 
topological materials, which should improve applications that require one-way propagation.

\section{ACKNOWLEDGMENTS}

The authors would like to thank X. Ni, X. C. Sun, and X. J. Zhang for stimulating discussions. The research reported in this publication was supported by funding from King Abdullah University of Science and Technology (KAUST).

\section{APPENDIX EFFECTIVE HAMILTONIAN WITH AIRFLOW}

The Hamiltonian with the airflow under the basis of $|d\rangle$, $\left|p_{+}\right\rangle$, and $\left|p_{-}\right\rangle$is expressed as follows [the same as Eq. (5)],

$$
H=\left[\begin{array}{ccc}
E_{d}(\vec{k}), & \sqrt{2} t_{x}^{12}\left(\sin k_{x} a-i \sin k_{y} a\right), & -\sqrt{2} t_{x}^{12}\left(\sin k_{x} a+i \sin k_{y} a\right), \\
\sqrt{2} t_{x}^{12}\left(\sin k_{x} a+i \sin k_{x} a\right), & \frac{1}{2}\left[E_{p y}(\vec{k})+E_{p x}(\vec{k})\right]+\Delta z, & \frac{1}{2}\left[E_{p y}(\vec{k})-E_{p x}(\vec{k})\right], \\
-\sqrt{2} i t_{x}^{12}\left(\sin k_{x} a-i \sin k_{y} a\right), & \frac{1}{2}\left[E_{p y}(\vec{k})-E_{p x}(\vec{k})\right], & \frac{1}{2}\left[E_{p y}(\vec{k})+E_{p x}(\vec{k})\right]-\Delta z
\end{array}\right],
$$

where $E_{d}(\vec{k})=E_{d}+2 t_{x}^{11}\left(\cos k_{x} a+\cos k_{y} a\right), E_{p y}(\vec{k})=E_{p y}+$ $2 t_{x}^{22} \cos k_{y} a+2 t_{y}^{22} \cos k_{x} a, E_{p x}(\vec{k})=E_{p x}+2 t_{x}^{22} \cos k_{x} a+$ $2 t_{y}^{22} \cos k_{y} a$. This Hamiltonian is sufficient to predict the topological invariant of the band gap and the topological transition by modulating the width of the rectangular waveguide and external velocity field. For illustration purposes, we choose the case presented in Fig. 1(c) as the starting point, in which introducing a weak airflow does not lead to a topologically nontrivial band gap, while if the airflow becomes stronger, topological transition occurs. In this case, $E_{p x}(0)=E_{p y}(0)<E_{d}(0)$ without the airflow. When the airflow is introduced, it is possible to achieve $E_{p x}(0)-\Delta z<E_{p y}(0)+\Delta z \approx E_{d}(0)$, indicating it is legitimate to employ a perturbation theory to project the $\left|p_{-}\right\rangle$state out and to get the effect Hamiltonian under the basis of $|d\rangle$ and $\left|p_{+}\right\rangle$.

We write $H$ as $H=\left[\begin{array}{cc}H_{A} & H_{A B} \\ H_{A B}^{\dagger} & H_{B}\end{array}\right]$ with an eigenvalue problem $\left[\begin{array}{cc}H_{A} & H_{A B} \\ H_{A B}^{\dagger} & H_{B}\end{array}\right]\left(\begin{array}{c}\phi_{A} \\ \phi_{B}\end{array}\right)=E\left(\begin{array}{c}\phi_{A} \\ \phi_{B}\end{array}\right)$ to be solved. We are interested in getting an effective $H_{A \text {,eff }}$ such that $H_{A, \text { eff }} \phi_{A}=E \phi_{A}$. To do this, we can replace $\phi_{B}$ with an expression of $\phi_{A}$, which is $\phi_{B}=\left(E-H_{B}\right)^{-1} H_{A B}^{\dagger} \phi_{A}$ and obtain $H_{A \text {,eff }}=H_{A}+H_{A B}\left(E-H_{B}\right)^{-1} H_{A B}^{\dagger}$.

In our case, we have

$$
\begin{aligned}
& H_{A}=\left[\begin{array}{cc}
E_{d}(\vec{k}), & \sqrt{2} t_{x}^{12}\left(\sin k_{x} a-i \sin k_{y} a\right), \\
\sqrt{2} t_{x}^{12}\left(\sin k_{x} a+i \sin k_{x} a\right), & \frac{1}{2}\left[E_{p y}(\vec{k})+E_{p x}(\vec{k})\right]+\Delta z
\end{array}\right], \\
& H_{A B}=\left[\begin{array}{c}
-\sqrt{2} t_{x}^{12}\left(\sin k_{x} a+i \sin k_{y} a\right), \\
\frac{1}{2}\left[E_{p y}(\vec{k})-E_{p x}(\vec{k})\right]
\end{array}\right], \\
& H_{B}=\frac{1}{2}\left[E_{p y}(\vec{k})+E_{p x}(\vec{k})\right]-\Delta z .
\end{aligned}
$$

In the spirit of perturbation, if the eigenvalue of $H_{A}$ differs much from that of $H_{B}$, we can approximate the eigenvalue $E$ of $H_{A \text {,eff }}$ by the zero-order eigenvalue of $H_{A}$. Then $\left(E-H_{B}\right)^{-1}$ can be approximated as $1 /(2 \Delta z)$, and

$$
H_{A B} H_{A B}^{\dagger}=\left[\begin{array}{cc}
2\left(t_{x}^{12}\right)^{2}\left[\sin ^{2}\left(k_{x} a\right)+\sin ^{2}\left(k_{y} a\right)\right], & -\frac{\sqrt{2}}{2} t_{x}^{12}\left(\sin k_{x} a+i \sin k_{y} a\right)\left[E_{p y}(\vec{k})-E_{p x}(\vec{k})\right], \\
-\frac{\sqrt{2}}{2} t_{x}^{12}\left(\sin k_{x} a-i \sin k_{y} a\right)\left[E_{p y}(\vec{k})-E_{p x}(\vec{k})\right], & \frac{1}{4}\left[E_{p y}(\vec{k})-E_{p x}(\vec{k})\right]^{2}
\end{array}\right] .
$$

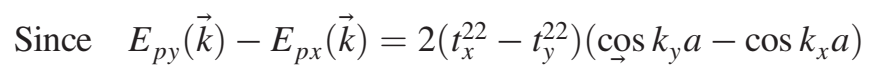
corresponds to high-order term of $\vec{k}$ for the case $k_{x} \approx k_{y} \approx 0$, all four elements of matrix $H_{A B} H_{A B}^{\dagger}$ correspond to second-order perturbation and can be neglected. Thus, the effective $H_{A \text {,eff }}$ is just $H_{A}$ and can be regarded as a Dirac equation in the region $k_{x} \approx k_{y} \approx 0$, which is written as 


$$
H_{A}=\left[\begin{array}{cc}
E_{d}(0), & \sqrt{2} t_{x}^{12}\left(k_{x} a-i k_{y} a\right), \\
\sqrt{2} t_{x}^{12}\left(k_{x} a+i k_{x} a\right), & \frac{1}{2}\left[E_{p y}(0)+E_{p x}(0)\right]+\Delta z
\end{array}\right],
$$

The new Hamiltonian (A2) is exactly the same as the wellknown QAH model, and there exists a topological transition point when $\left[E_{p y}(0)+E_{p x}(0)\right] / 2+\Delta z=E_{d}(0)$, which is also confirmed by the numerical calculation from both Eqs. (A1) and (A2).

[1] M. Z. Hasan and C. L. Kane, Topological insulators, Rev. Mod. Phys. 82, 3045 (2010).

[2] X. L. Qi and S.C. Zhang, Topological insulators and superconductors, Rev. Mod. Phys. 83, 1057 (2011).

[3] J. E. Moore, The birth of topological insulators, Nature (London) 464, 194 (2010).

[4] M. Onoda, S. Murakami, and N. Nagaosa, Hall Effect of Light, Phys. Rev. Lett. 93, 083901 (2004).

[5] F. D. M. Haldane and S. Raghu, Possible Realization of Directional Optical Waveguides in Photonic Crystals with Broken Time-Reversal Symmetry, Phys. Rev. Lett. 100, 013904 (2008).

[6] Z. Wang, Y. D. Chong, J. D. Joannopoulos, and M. Soljačić, Reflection-Free One-Way Edge Modes in a Gyromagnetic Photonic Crystal, Phys. Rev. Lett. 100, 013905 (2008).

[7] M. C. Rechtsman, J. M. Zeuner, Y. Plotnik, Y. Lumer, D. Podolsky, F. Dreisow, S. Nolte, M. Segev, and A. Szameit, Photonic Floquet topological insulators, Nature (London) 496, 196 (2013).

[8] L. Lu, J. D. Joannopoulos, and M. Soljacic, Topological photonics, Nat. Photonics 8, 821 (2014).

[9] Z. Yang, F. Gao, X. Shi, X. Lin, Z. Gao, Y. Chong, and B. Zhang, Topological Acoustics, Phys. Rev. Lett. 114, 114301 (2015).

[10] X. Ni, C. He, X. C. Sun, X. P. Liu, M. H. Lu, L. Feng, and Y. F. Chen, Topologically protected one-way edge mode in networks of acoustic resonators with circulating air flow, New J. Phys. 17, 053016 (2015).

[11] M. Xiao, G. Ma, Z. Yang, P. Sheng, Z. Q. Zhang, and C. T. Chan, Geometric phase and band inversion in periodic acoustic systems, Nat. Phys. 11, 240 (2015).

[12] P. Wang, L. Lu, and K. Bertoldi, Topological Phononic Crystals with One-Way Elastic Edge Waves, Phys. Rev. Lett. 115, 104302 (2015).

[13] V. Peano, C. Brendel, M. Schmidt, and F. Marquardt, Topological Phases of Sound and Light, Phys. Rev. X 5, 031011 (2015).

[14] A. B. Khanikaev, R. Fleury, S. H. Mousavi, and A. Alu, Topologically robust sound propagation in an angularmomentum-biased graphene-like resonator lattice, Nat. Commun. 6, 8260 (2015).
[15] M. Xiao, Z. Q. Zhang, and C. T. Chan, Surface Impedance and Bulk Band Geometric Phases in One-Dimensional Systems, Phys. Rev. X 4, 021017 (2014).

[16] M. Xiao, W. J. Chen, W. Y. He, and C. T. Chan, Synthetic gauge flux and Weyl points in acoustic systems, Nat. Phys. 11, 920 (2015).

[17] S. H. Mousavi, A. B. Khanikaev, and Z. Wang, Topologically protected elastic waves in phononic metamaterials, Nat. Commun. 6, 8682 (2015).

[18] E. Prodan and C. Prodan, Topological Phonon Modes and Their Role in Dynamic Instability of Microtubules, Phys. Rev. Lett. 103, 248101 (2009).

[19] N. Swinteck, S. Matsuo, K. Runge, J. O. Vasseur, P. Lucas, and P. A. Deymier, Bulk elastic waves with unidirectional backscattering-immune topological states in a timedependent superlattice, J. Appl. Phys. 118, 063103 (2015).

[20] R. Süsstrunk and S. D. Huber, Observation of phononic helical edge states in a mechanical topological insulator, Science 349, 47 (2015).

[21] C. L. Kane and T. C. Lubensky, Topological boundary modes in isostatic lattices, Nat. Phys. 10, 39 (2014).

[22] J. Paulose, B. G.-g. Chen, and V. Vitelli, Topological modes bound to dislocations in mechanical metamaterials, Nat. Phys. 11, 153 (2015).

[23] R. Fleury, A. B. Khanikaev, and A. Alù, Floquet topological insulators for sound, arXiv:1511.08427.

[24] J. Lu, C. Qiu, S. Xu, Y. Ye, M. Ke, and Z. Liu, Dirac cones in two-dimensional artificial crystals for classical waves, Phys. Rev. B 89, 134302 (2014).

[25] C. L. Fefferman, J. P. Lee-thorp, and M. I. Weinstein, Edge states in honeycomb structures arXiv:1506.06111v2.

[26] X. Huang, Y. Lai, Z. H. Hang, H. Zheng, and C. T. Chan, Dirac cones induced by accidental degeneracy in photonic crystals and zero-refractive-index materials, Nat. Mater. 10, 582 (2011).

[27] J. Mei, Y. Wu, C. T. Chan, and Z. Q. Zhang, First-principles study of Dirac and Dirac-like cones in phononic and photonic crystals, Phys. Rev. B 86, 035141 (2012).

[28] X.-L. Qi, Y.-S. Wu, and S.-C. Zhang, Topological quantization of the spin Hall effect in two-dimensional paramagnetic semiconductors, Phys. Rev. B 74, 085308 (2006).

[29] L. M. Brekhovskikh and I. U. P. Lysanov, Fundamentals of Ocean Acoustics (Springer, New York, 2003).

[30] B. A. Bernevig, T. L. Hughes, and S. C. Zhang, Quantum spin Hall effect and topological phase transition in $\mathrm{HgTe}$ quantum wells, Science 314, 1757 (2006).

[31] K. Sakoda, Dirac cone in two- and three-dimensional metamaterials, Opt. Express 20, 3898 (2012).

[32] R. Fleury, D. L. Sounas, C. F. Sieck, M. R. Haberman, and A. Alù, Sound isolation and giant linear nonreciprocity in a compact acoustic circulator, Science 343, 516 (2014).

[33] Y. D. Chong, X. G. Wen, and M. Soljačić, Effective theory of quadratic degeneracies, Phys. Rev. B 77, 235125 (2008).

[34] C. Fang, M. J. Gilbert, and B. A. Bernevig, Bulk topological invariants in noninteracting point group symmetric insulators, Phys. Rev. B 86, 115112 (2012). 